\title{
Performance Comparison of VoIP Codecs on Multiple Operating Systems using IPv4 and IPv6
}

\author{
Hira Sathu and Mohib A. Shah
}

\begin{abstract}
In this paper, the performance of Voice over Internet Protocol (VoIP) for five different codecs, using IPv4 and IPv6 is established. The codecs tested were G.711.1, G.711.2, G.723.1, G.729.2 and the G.729.3 codec. The operating systems used were; Windows XP, Windows Vista and the Windows 7. Parameters considered for these performance tests were RTT (round trip time), jitter, and throughput. The results arrived at aid a user organisation of VoIP with the preferred choice of the IP version stack to be used for a given operating system. Likewise, where a user is constrained to adopt a given IP stack, the codec that should be used over different Windows OSs available on the systems is also identifiable.
\end{abstract}

Index Terms-VoIP, performance analysis, codec, IPv4, IPv6, Windows XP, Windows Vista and Windows 7.

\section{INTRODUCTION}

Larger number of desktop, laptop and other computing machines now require IP addresses for accessing the internet and networking than ever before. It is anticipated that all available IP addresses in IPv4 will be exhausted in next few years. IPv6 was designed by the Internet Engineering Task Force (IETF) to be the successor of IPv4. The main advantages of IPv6 is its ability to support large numbers of addresses, 2128 IP addresses (128-bit address field in IPv6 packet) with no longer a need for Network address translation (NAT). Future communications over IPv6 would lead to improved voice and video application experience on account of lower latency on account of communications without NAT.

VoIP (Voice over Internet Protocol) is designed to provide voice communications over packet switched networks such as the Internet. VoIP networks use a range of protocols and audio codecs to control communication. These protocols cover the connection, set-up and tear-down of calls, while the codecs encode the speech so that it can be transported over the internet. The main reason VoIP has become so popular over the last few years is because of the reduced cost associated with using VoIP compared to the PSTN (Public Switched Telephone Network). As VoIP gains greater popularity and soft phones are being introduced in larger numbers, performance of operating systems may reduce the quality of VoIP. To ascertain this authors have carried out experiments to identify the impact of different operating

Manuscript received October 9, 2011; revised March 5, 2012.

H. Sathu is a Principal Academic staff member at Unitec Institute of Technology, Auckland, New Zealand (e-mail: hsathu@unitec.ac.nz).

M. A. Shah was a student at Unitec Institute of Technology, Auckland New Zealand. He is now tutoring at Unitec, New Zealand (e-mail: mshah.itexpert@gmail.commshah@unitec.ac.nz). systems on VoIP codecs over both IPv4 and IPv6 environments.

Multiple Microsoft operating systems were involved in this experiment to measure the best operating system performance for VoIP. Thus we have selected Windows XP, Windows Vista and Windows 7 operating systems. In this study, the performance of VoIP (Voice over Internet Protocol) codecs is tested on Windows XP, Windows Vista and Windows 7 and their results are compared for both IPv4 and IPv6 networks.

In this study, the performance of VoIP (Voice over Internet Protocol) codecs is tested on Windows XP, Windows Vista and Windows 7 and their results are compared for both IPv4 and IPv6 networks.

In [1], several ITU-T (International Telecommunications Union) standard voice codec algorithms known as G.711.1, G.723.1, and G.729 are compared. Each codec has its own speed, frame size, delay and payload as shown in Table 1 below.

TABLE I: Multiple VoIP CODECS AND THEIR FrAME Sizes [1]

\begin{tabular}{|c|c|c|c|}
\hline Codec & G.711 & G.723.1 & G.729 \\
\hline Coding speed (Kbps) & 64 & $5.3 / 6.3$ & 8 \\
\hline Frame size (ms) & 20 & 30 & 10 \\
\hline Processing Delay (ms) & 20 & 30 & 10 \\
\hline Lookahead Delay (ms) & 0 & 7.5 & 5 \\
\hline DSP MIPS & 0.34 & 16 & 20 \\
\hline Payload (bytes) & 160 & $20 / 24$ & 20 \\
\hline Number of flows & 7 & $84 / 71$ & 56 \\
\hline $\begin{array}{c}\text { Subscribed Rate packet time } \\
\text { (ms) }\end{array}$ & 20 & $30.2 / 30.5$ & 20 \\
\hline
\end{tabular}

The organisation of this paper is in six sections. The next section covers related works and the contribution this paper makes, Section 3 includes information regarding network set up for the current study, and Section 4 covers the traffic generating tool description. Section 5 outlines the results of the experiment and the last (sixth) section concludes the study and is followed by the references and Table 3 relating to throughput Comparison of IPv4 and IPv6 using different Windows OS.

\section{RELATED WORK}

In [2], the authors compared the performance of VoIP network using SIP (Session Initiation Protocol) over both IPv4 and IPv6 networks. They tested performance of VoIP using IPv4 and IPv6 as well as usage of IPv6to4 tunnelling and Teredo tunnelling. They concluded that the native IPv4 had slightly less delay than the native IPv6 and IPv6to4 tunnelling had much less delay than the Teredo tunnelling. In 
[3], the authors discussed the connection of IPv6 domains using the current IPv4 network without setting up an explicit tunnel between the two connected domains. The report explains the use of the IPv6to4 pseudo-interface, which is when the IPv6 packet is encapsulated in an IPv4 packet at one end, and is then sent over the IPv4 cloud. When the packet reaches the other end, it is then unpacked. The authors in [4] compared different aspects of VoIP between IPv4 and IPv6. The authors considered jitter, delay, packet loss and throughput on different systems using $0-200 \mathrm{Mbps}$ traffic. The authors showed that for Windows XP, the average delay between packets for IPv4 and IPv6 is almost the same, except at $100 \mathrm{Mbps}$ when IPv6 delay is approximately $0.002 \mathrm{~ms}$ more than IPv4. From $0-50 \mathrm{Mbps}$ of traffic, packet loss was equal between the two IP versions, at 0 lost packets, but for 100, 150 and $200 \mathrm{Mbps}$, IPv6 packet loss rose to 4, 13, and 17 packets lost respectively, whereas IPv4 rose to 0,12 and 17 packets lost respectively. The average jitter reduced fairly consistently up to $100 \mathrm{Mbps}$, but from $100 \mathrm{Mbps}$ to $200 \mathrm{Mbps}$ IPv4 showed less jitter than IPv6 by $0.05 \mathrm{~ms}$. Overall, IPv4 had better performance across the tests compared to IPv6.

In [5], authors discussed the effect of speech and audio compression on speech recognition performance. The authors investigated GSM (Global System for Mobile Communications) full rate, G.711, G.723.1 and MPEG coders and found out that MPEG transcoding degrades the speech recognition performance for low bitrates and maintains the performance of specialised speech coders like GSM or G.711.

In [6] experiment was conducted by researchers to test the performance of VoIP with IPv4 and IPv6 using IPv6to4 tunnelling and NAT (Network Address Transition) mechanism to identify the delta, jitter, packet loss, MOS (Mean Opinion Score) and throughput. Their results demonstrate that quality of VoIP is impacted due to transition mechanism; however voice quality decreases as network traffic capacity is increased.

To date (early 2010) no one has compared the performance of Windows XP, Windows Vista and Windows 7 using VoIP codecs on IPv4 and IPv6. The contribution of this paper is to compare the performance of above codecs with Windows XP, Windows Vista and Windows 7 operating systems over both IPv4 and IPv6 networks.

\section{NETWORK SETUP}

The proposed network test-bed was setup based on two different configurations with IPv4 and IPv6. The first setup was based on the IPv4 configuration, where a workstation is connected to another workstation via a router. Second setup configuration was based on IPv6 network where two workstations were connected through a router. In this experiment standard Category 5e cables was used in both networks.

To configure IPv4 network, two different workstations were used with identical hardware and software. Each network included two workstations using Microsoft Windows XP, Vista and Windows 7 operating systems. A workstation wired connected to a router using IPv4 address and RIP 2 routing protocols. The other side of the router was wired connected to second workstation with same setting except subnet. Fig. 1 below illustrates the network setup.

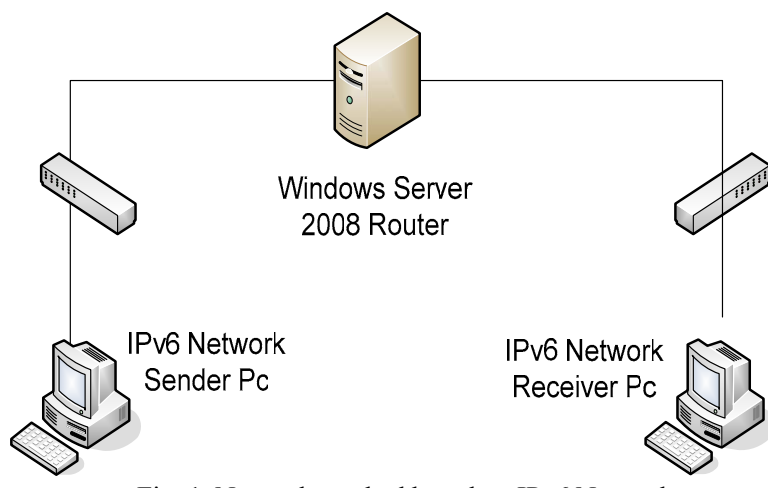

Fig. 1. Network test bed based on IPv6 Network.

This experiment was conducted in order to evaluate the performance of all IPv4 network and IPv6 network with different operating systems. Parameters calculated were RTT, jitter and throughput and compared against IPv6 and IPv4 networks over different Windows operating systems.

The hardware benchmark comprised of an Intel ${ }^{\circledR}$ Core $^{\mathrm{TM}} 2$ Duo $63001.87 \mathrm{GHz}$ processor with 2.00 GB RAM for the efficient operation of Windows 7 and Windows XP, an Intel Pro/100 S Desktop Adapter NIC and a Western Digital Caviar SE $160 \mathrm{~GB}$ hard-drive on the two workstations. In order to make comparisons, we used identical hardware for all our tests. A benchmarking tool known as CPU-Z was used to determine if all computers were identical. Two routers, two Switches and Cat5e fast Ethernet cables were also used for creating the test-bed.

\section{Data Generation And Traffic Measurement Tool}

D-ITG (Distributed Internet Traffic Generator) [7] was the tool that was selected to generate and measure the traffic. This tool was the one selected as it could support both IPv4 and IPv6 traffic and worked across a range of operating systems including Windows XP, Windows Vista and Windows 7. D-ITG tool was designed with fixed frame size and packets per seconds for each VoIP codec as visible in the Table 2 below:

TABLE II: D-ITG CODECS FOR VOIP PACKET GENERATOR [7]

\begin{tabular}{|c|c|c|c|}
\hline Codecs & Samples & Framesize & Packets (per sec) \\
\hline G.711.1 & 1 & 80 & 100 \\
\hline G.711.2 & 2 & 80 & 50 \\
\hline G.729.2 & 2 & 10 & 50 \\
\hline G.729.3 & 3 & 10 & 33 \\
\hline G.723.1 & 1 & 30 & 26 \\
\hline
\end{tabular}

D-ITG command mode version was installed on both networks to send and receive VoIP traffic. D-ITG sender was installed on a workstation and D-ITG receiver was installed on another workstation. The experiments comprised of performing 10 flows (simultaneously) with over 10 number of runs for every codec type, on every operating system, for IPv4 and IPv6. A flow contains 1000 packets of a codec and (is equivalent to a VoIP call) sending from one workstation to another. A script was used to send 10 flows at the same time and average results were obtained. The number of runs is continued until $90 \%$ confidence interval in results is achieved. Each codec has its own standard packet size, which effects the results obtained (Table 2 ).

The RTT (Round Trip Time), throughput and jitter were measured for both IPv4 and IPv6 on Windows XP, Windows 
Vista and Windows 7, and for the G.711.1, G.711.2, G.723.1, G.729.2, and G.729.3 codecs over a fast Ethernet VoIP network as shown in the network test bed diagram (Figure 1) above.

\section{RESUlts}

The results for comparing the performance of VoIP codecs on different operating systems using the IPv4 and IPv6 protocols are depicted in the figures below with a brief explanation of the results. The results are presented in the sequence: RTT, Jitter and last of all the throughput.

\section{A. Experiment 1: RTT}

The RTT comparison for the OSs Windows XP, Windows Vista and Windows 7 shows that overall the RTT times was lower for IPv4 than IPv6 while using Windows XP OS (Figure 2) for all the codecs being tested.

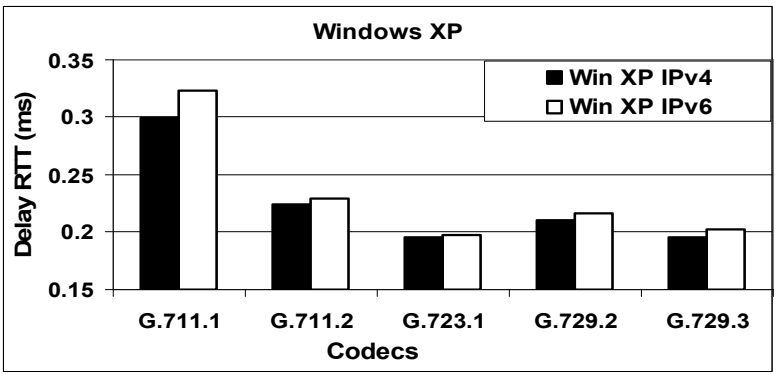

Fig. 2. RTT Comparison for IPv4 and on windows XP.

Over Windows Vista, RTT performance for IPv6 was better for the codecs G711.1, G729.2 and G729.3.For the codecs G711.2, G723.1, IPv4 was lower hence better.Figure 3 below depicts the details for this.

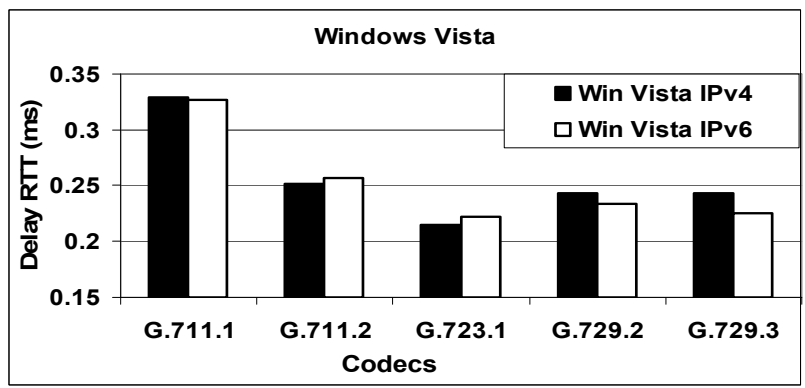

Fig. 3. RTT Comparison for IPv4 and on windows vista.

Over Windows 7 OS the best performance was of G723.1 codec using IPv6. for all other Codecs IPv4 performance was better than IPv6, while codec G729.2 performance for both was about the same. Figure 4 below depicts the details for this.

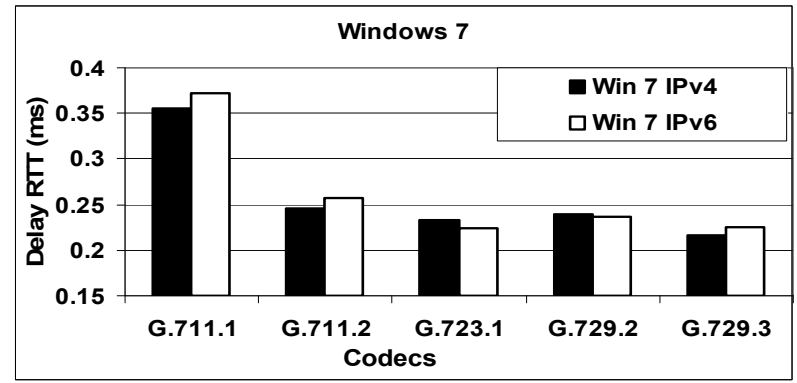

Fig. 4. RTT Comparison for IPv4 and on windows 7.

\section{B. Experiment 2: Jitter.}

As for the jitter tests over Windows XP all codecs except G711.1 performance of IPv6 was better than IPv4. Figure 5 below depicts the details for this.

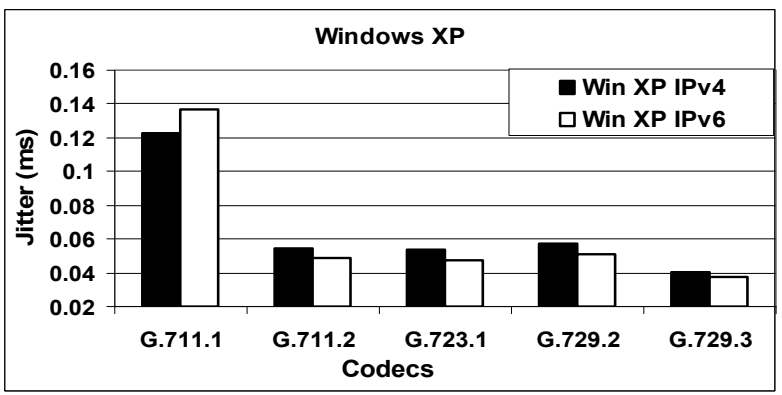

Fig. 5. Jitter Comparison for IPv4 and IPv6 on windows XP.

Over Windows Vista IPv6 performance was significantly better than IPv4 for G729.2 codec and marginally better for G729.3 codec. For the remaining codecs G711.1, G711.2 and G723.1 IPv4 performance was better. Figure 6 below depicts the details for this

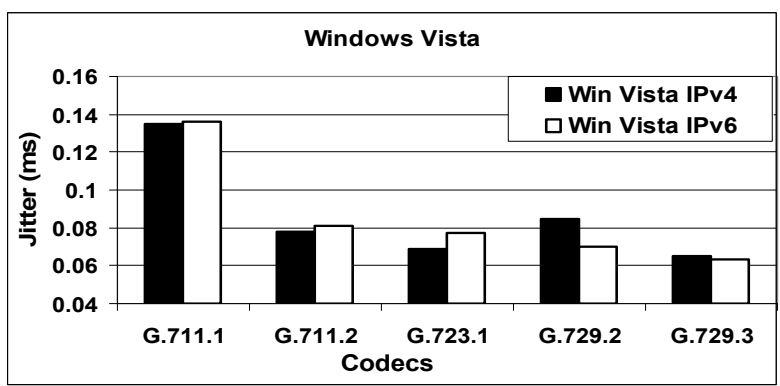

Fig. 6. Jitter Comparison for IPv4 and IPv6 on windows vista.

Over Windows 7 IPv6 performance was better while using codecs G723.1 and G729.2. For all other codecs the performance was better for IPv4. Figure 7 below depicts the details for this.

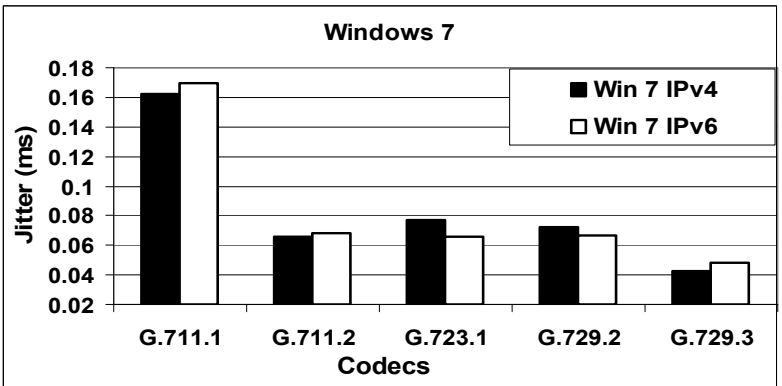

Fig. 7. Jitter Comparison for IPv4 and IPv6 on windows 7.

\section{Experiment 3: Throughput}

The details for this experiment have been considered codec wise as below:

For codec G711.1 both Windows XP and Windows Vista OSs, IPv6 provided better throughput $(689.727 \mathrm{Kbps}$ and $684.684 \mathrm{Kbps}$ ) than over IPv4. Over Windows 7 OS, IPv4 throughput was only marginally better than IPv6 being $687.85 \mathrm{Kbps}$ and $687.307 \mathrm{Kbps}$ respectively.

For codec G711.2, Windows 7 OS provided the best performance (657.99Kbps) using IPv6 even though marginally better than IPv4 at $657.95 \mathrm{Kbps}$. However, IPv4 
performed better than IPv6 over the Windows XP and Windows Vista OSs.

For codec G723.1, usage of IPv4 protocol consistently provided better throughput than IPv6 protocol over all versions of Windows OSs.

For codec G729.2, IPv6 scored better than IPv4 over Windows XP and Windows 7, while over Windows Vista IPv4 was marginally better than IPv6.

For codec G729.3, IPv6 fared better than IPv4 over Windows XP. Whereas, IPv4 fared better than IPv6 even though marginally over Windows Vista and Windows 7 OSs. The throughput details for all the codecs tested are presented at TABLE III below.

\begin{tabular}{|c|c|c|c|c|c|c|}
\hline \multirow[b]{2}{*}{$\begin{array}{c}\text { Cod } \\
\text { ec } \\
\text { Type }\end{array}$} & \multicolumn{6}{|c|}{ Throughput (Kbps) } \\
\hline & $\begin{array}{c}\text { Win } \\
\text { XP } \\
\text { IPv4 }\end{array}$ & $\begin{array}{c}\text { Win } \\
\text { XP } \\
\text { IPv6 }\end{array}$ & $\begin{array}{c}\text { Win } \\
\text { Vista } \\
\text { IPv4 }\end{array}$ & $\begin{array}{c}\text { Win } \\
\text { Vista } \\
\text { IPv6 }\end{array}$ & $\begin{array}{l}\text { Win } 7 \\
\text { IPv4 }\end{array}$ & $\begin{array}{l}\text { Win } 7 \\
\text { IPv6 }\end{array}$ \\
\hline $\begin{array}{c}\text { G.71 } \\
1.1\end{array}$ & $\begin{array}{c}681.3 \\
61266 \\
1 \\
\end{array}$ & $\begin{array}{l}689.72 \\
71231\end{array}$ & $\begin{array}{c}683.349 \\
3567\end{array}$ & $\begin{array}{c}684.684 \\
2507\end{array}$ & $\begin{array}{r}687.85 \\
69308\end{array}$ & $\begin{array}{c}687.3070 \\
925\end{array}$ \\
\hline $\begin{array}{c}\text { G. } 71 \\
1.2\end{array}$ & $\begin{array}{c}655.3 \\
02733 \\
4\end{array}$ & $\begin{array}{l}654.12 \\
25674\end{array}$ & $\begin{array}{c}653.464 \\
8286\end{array}$ & $\begin{array}{c}653.096 \\
8708\end{array}$ & $\begin{array}{c}657.95 \\
6126\end{array}$ & $\begin{array}{c}657.9966 \\
562\end{array}$ \\
\hline $\begin{array}{c}\text { G.72 } \\
3.1 \\
\end{array}$ & $\begin{array}{l}77.40 \\
19337 \\
\end{array}$ & $\begin{array}{c}77.328 \\
3562 \\
\end{array}$ & $\begin{array}{c}77.1079 \\
412\end{array}$ & $\begin{array}{c}76.9556 \\
766\end{array}$ & $\begin{array}{c}77.510 \\
6225 \\
\end{array}$ & $\begin{array}{c}77.48777 \\
35 \\
\end{array}$ \\
\hline $\begin{array}{c}\text { G.72 } \\
9.2\end{array}$ & $\begin{array}{c}108.8 \\
40437 \\
5\end{array}$ & $\begin{array}{l}109.56 \\
30361\end{array}$ & $\begin{array}{c}108.997 \\
4913\end{array}$ & $\begin{array}{c}108.984 \\
4235\end{array}$ & $\begin{array}{l}109.50 \\
19974\end{array}$ & $\begin{array}{c}109.5523 \\
345\end{array}$ \\
\hline $\begin{array}{c}\text { G.72 } \\
9.3\end{array}$ & $\begin{array}{l}97.70 \\
20266\end{array}$ & $\begin{array}{c}98.166 \\
9153\end{array}$ & $\begin{array}{c}97.7703 \\
485\end{array}$ & $\begin{array}{c}97.7089 \\
436\end{array}$ & $\begin{array}{c}98.329 \\
3808\end{array}$ & $\begin{array}{c}98.23930 \\
14\end{array}$ \\
\hline
\end{tabular}

\section{CONCLUSION}

The above results reveal a multitude of combinations for optimal performance of VoIP. The results arrived at aid a user organisation of VoIP with the preferred choice of the IP version stack to be used for a given operating system. Likewise where a user is constrained to adopt a given IP stack, the preferred codec could be used for different Windows OSs to obtain the best performance.

\section{ACKNOWLEDGMENTS}

We would like to show appreciation to UNITEC, Institute of Technology for supporting the research team and providing us this opportunity to complete this research.

\section{REFERENCES}

[1] J. K. Muppala, T. Bancherdvanich, and A. Tyagi. "VoIP Performance on Differentiated Services Enabled Network," (ICON 2000). Proceedings. IEEE International Conference on Networks, pp. 419-423, 2000.

[2] T. Hoeher, M. Petraschek, S. Tomic, and M. Hirschbichler. "Evaluating Performance Characteristics of SIP over IPv6," IEEE Journal of Networks, vol. 2, no. 4, pp. 10, 2007.

[3] B. Carpenter and K. Moore. "Connection of IPv6 Domains via IPv4 Clouds," Internet proposed standard RFC 3056, 2001.

[4] R. Yasinovskyy, A. L. Wijesinha, R. K. Karne, and G. Khaksari. "A Comparison of VoIP Performance on IPv6 and IPv4 Networks," IEEE/ACS International Conference on Computer Systems and Applications, pp. 603-609, 2009.

[5] L. Besacier, C. Bergamini, D. Vaufreydaz, and E. Castelli. "The effect of speech and audio compression on speech recognition performance," Multimedia Signal Processing, IEEE Fourth Workshop on 2001. pp. 301-306, August 2002.

[6] R. Yasinovskyy, A. L. Wijesinha, and R. Karne. "Impact of IPSec and 6to4 on VoIP Quality over IPv6," IEEE International Conference on 10th, Telecommunications, ConTEL, pp. 235-242, 2009.

[7] B. Alessio, D. Alberto, and P. Antonio, "Multi-Protocol and Multi-Platform Traffic Generation and Measurement," Available: http://www.grid.unina.it/software/ITG/, 2009.

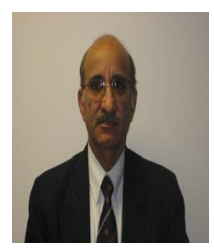

Hira Sathu is a Principal Academic staff member at Unitec Institute of Technology, Auckland, New Zealand for over 17 years. Prior to his academic roles at Unitec he worked for the House of Siemens, Germany (4 years) and the Department of Defence, India (20 years). Of recent he has been an invited speaker at International Conferences, NZ Info Security Forum and helped chair sessions at various National and International conferences.

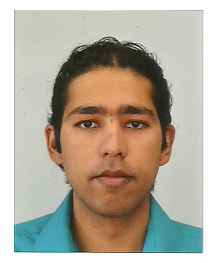

Mohib A. Shah recently completed his Master's degree in Computing and he is currently tutoring at Unitec, New Zealand. $\mathrm{He}$ is interested in NGN activities such as VoIP, VVoIP, Wireless Network and Mobile communication based on IPv6 infrastructure. He received a BCS degree and a Post Graduate Dip in Information Technology from Unitec, New Zealand. 